\title{
Oscillating Universe with a Quantized Black Hole
}

\author{
Sascha Kulas \\ sascha.kulas@gmx.net
}

\begin{abstract}
In cosmology dark energy and dark matter are included in the $\Lambda$ CDM model, but they are still completely unknown. On the other hand the trans-Planckian problem leads to unlikely high photon energies for black holes. We introduce a model with quantized black hole matter. This minimizes the transPlanckian problem extremely and leads to a scalar field in the oscillating universe model. We show that the scalar field has the same characteristics as a vacuum energy field and leads to the same Casimir effect. Shortly after the beginning of the big bounce this field decays locally and leads to the production of dark matter. In this model no inflation theory is needed. We emphasize that this model is mainly a phenomenological approach with the aim of new impetus to the discussion.
\end{abstract}

Keywords: Oscillating universe; big bang; big bounce; Hubble constant; dark energy; dark matter; inflation; vacuum energy density; Casimir effect. PACS Nos.: 98.80.-k

\section{Introduction}

The $\Lambda$ CDM model is very successful in describing the universe from the early beginning (assuming a big bang) and compatible to the CMB in a lot of details. In this model the scenario of an inflation phase is included, that solves the three main problems: the flatness problem, the horizon problem and the problem of magnetic monopoles. Since it has some other problems, e.g. the entropy is not growing during expansion, it motivates for other views. One other view is the scenario of a big bounce. That means at some point the expansion of a universe stops and it contracts again. Thus the big bang is the result of a collapse of a predecessor universe.

We look at a similar scenario where the predecessor universe ends up in a black hole. By assuming a mass quantization of black holes, the effective 
speed of light of photons around a black hole due to Hawking radiation is much lower than the speed of light $c_{0}$ in vacuum without strong gravitational fields and this leads in combination with the evaporation of black holes to a stable scalar field. After evaporating down to the Planck scale the black hole explodes and a new universe is created, embedded in a scalar field of the predecessor universe.

Since we will use an effective speed of light and want to show the effect by this effective speed, we will use natural units in the following.

\section{The trans-Planckian problem of black holes and the scalar field}

The Schwarzschild radius $R_{S}$ of a black hole describes the outer boarder where even light cannot escape the gravitational attraction

$$
R_{S}=\frac{2 G M}{c_{0}^{2}}
$$

Here $G$ is the gravitational constant and $M$ the mass of the black hole. $c_{0}$ is the speed of light in vacuum. Now we quantize the mass of the black hole. This idea is not new $[(1),(2),(3),(15)]$. We write equation (1) in this form

$$
c_{0}=\sqrt{\frac{2 G M}{R_{S}{ }^{2}}}=\sqrt{\frac{2 G N M_{P}}{R_{S}{ }^{2}}}
$$

We use the Planck mass $M_{P}$ as the fundamental mass for black holes, since it can be shown that the smallest black hole would have the Planck mass [(4)]. The number $N$ is brought to the other side and we end up with

$$
\frac{c_{0}}{\sqrt{N}}=c\left(R_{S}\right)=\sqrt{\frac{2 G M_{P}}{R_{S}^{2}}}
$$

We define $\frac{c_{0}}{\sqrt{N}}$ as the effective speed of light $c\left(R_{S}\right)$ which is only dependent on the Schwarzschild radius on the right side. The other values are constants. This gets clearer when $\frac{c_{0}}{\sqrt{N}}$ is seen as an effective speed of light, similar to the speed of light in a medium with refractive index $n$. Then this reduced speed of light is only dependent on the Schwarzschild radius. Here $M_{P}=\sqrt{\frac{\hbar c_{0}}{G}}$ is the Planck mass with the reduced Planck's constant $\hbar$. 
Together with the value for the Planck mass and some transformations this leads to

$$
c\left(R_{S}\right)=c_{0} \sqrt{\frac{2 L_{P}}{R_{S}}}
$$

Here $L_{P}$ is the Planck length. That means a bigger Schwarzschild radius leads to a smaller speed of light. This is comprehensible because in a sphere with a bigger radius the photons interact on longer time scales with the refractive medium. In a black hole of the size of the Planck length (created by a Planck mass) the photons do not reduce their speed of light, because the black hole is that small.

In the following we are interested in the evaporation of black holes and Hawking radiation $[(5)]$. A new publication $[(6)]$ shows one way how to change the black hole information paradox to a black hole information puzzle. When we assume the entanglement of photons [(14)], the effective speed of light is induced to photons leaving the black hole in the following way: First the created photon pairs near the black hole are split. One photon leaves the black hole while the other enters it by crossing the event horizon. This photon realizes the extreme gravitational force and in our model the refractive medium. Since both photons are entangled the other photon also realizes the reduced speed of light. In another more simple picture the refractive medium is not limited to the Schwarzschild radius $\left(r<R_{S}\right)$ and the medium is also around the black hole. The leaving photon still realizes the refractive medium by a reduced speed of light.

Due to the quite small speed of light for the photons of Hawking radiation (see Appendix A), the trans-Planckian problem for black holes can be strongly suppressed. Here field modes near the black hole horizon have arbitrarily high frequencies. This is shown by the frequency $f(t)$

$$
f(t)=f_{0} e^{k t}=f_{0} e^{\frac{c(t)}{2 R_{S}} t}
$$

Together with the equation for $c\left(R_{S}\right)$ this leads to an exponential factor which is strongly suppressed by $\sqrt{\frac{2 L_{P}}{R_{S}}}$, where $R_{S}$ is the Schwarzschild radius of a black hole.

Together with the effective speed of light we can derive an effective rest 
mass for the photons with energy $E$

$$
m_{e f f}=\frac{E}{c\left(R_{S}\right)^{2}}=\frac{E R_{S}}{2 L_{P} c_{0}^{2}}
$$

The black hole evaporates due to Hawking radiation with a Hawking temperature $T_{H}$

$$
T_{H}=\frac{\hbar c_{0}^{3}}{8 \pi G M k_{B}}
$$

Here $k_{B}$ the Boltzmann constant.

The photons gain a temperature $T_{H}$ and an energy $E=k_{B} T_{H}$. By adding this energy in equation (6), we end up with an effective mass of

$$
m_{e f f}=\frac{\hbar}{8 \pi c_{0} L_{P}}
$$

This mass is only defined by constants. It is not dependent on the Schwarzschild radius or the mass of the black hole anymore. That means during evaporation a scalar field around the black hole is created, that consists of particles with the mass $m_{e f f}=8.659757 \times 10^{-10} \mathrm{Kg}$. This applies $m_{e f f}=0.03979 \times M_{P}$ with $x=0.03979$. We calculate $x$ here because we need it for other calculations later.

The scalar field has interesting features and is very similar to the cosmological quintessence [(8)]. Since it is made of photons with an effective rest mass the individual speed of the particles is extremely low $\left(\approx 10^{-22} \mathrm{~m} / \mathrm{s}\right.$, see Appendix A). These photons do not interact with other particles of effective mass in the field in the first order because of their photonic properties.

Since their speed is so low, their energy density is not changing over time in the first approximation. Due to the characteristics of Hawking radiation, space is filled with these particles in an orderly pattern after the complete evaporation of the black hole.

When considering a perfect fluid, described by its density $\rho$ and pressure $p$ filling a Robertson Walker spacetime, the Friedmann equation for such a fluid is

$$
\dot{\rho}+\frac{3 \dot{a}}{a}\left(\rho+\frac{p}{c^{2}}\right)=0
$$

Here a dot means derivative with respect to time $t$. That means every change of the energy density is proportional to the sum of positive energy density and pressure. For our scalar field the change is approximately zero and thus 
the pressure is negative. That means normal matter which is surrounded by the effective mass particles realizes a repulsive force in addition to the attractive gravitational force of the normal matter itself.

\section{The Hubble constant}

When we consider an oscillating universe scenario the black hole with its orginal Schwarzschild radius $R_{S}$ in the previous universe evaporated and shrank until it reached the size of a planck length. An explosion of the black hole at this size is assumed [(9)], leading to a big bang embedded in the scalar field made of Hawking radiation. A sphere with the radius $R_{S}$ embodies the delimitation of this scalar field. Everything that was created during the big bang realizes this scalar field.

Since we use the sphere, we take a Newtonian ansatz on time scales much later than the Big Bang. That means we leave the Schwarzschild metric and use the Euclidian metric as a basis. Due to the low speed of the effective mass particles (see Appendix A) a Newtonian consideration makes sense here.

The differential equation for particles in a sphere with a uniform density distribution has the following form:

$$
\ddot{x}(t)=\frac{G M}{R_{S}{ }^{3}} x(t)
$$

By using the well-known ansatz $x(t)=x_{0} e^{t / \tau}$ this leads to $\tau=\sqrt{\frac{R_{S}^{3}}{G M}}$ ending up with a scalar factor $a(t)=e^{\sqrt{\frac{G \cdot M}{R_{S}^{3}}} t}$. The Hubble constant has the form

$$
H_{0}=\frac{\dot{a}(t)}{a(t)}=\sqrt{\frac{G M}{R_{S}{ }^{3}}}
$$

The pressure is negative and that means the Hubble constant has a positive value. This form of the Hubble constant will be used later.

In the next step it is important to know how big the sphere is. When the universe expands further, it will reach the outer shell of the sphere. Further in the future the mass inside the sphere looks like a point mass with the Schwarzschild radius $R_{S}=\frac{2 G M}{c_{0}^{2}}$ and the mass $M_{S}=\frac{R_{S} c_{0}^{2}}{2 G}$. The gravitational acceleration for normal mass on the shell has the form $g\left(r=R_{S}\right)=G \frac{M_{S}}{R_{S}{ }^{2}}$ and with the point mass this leads to $g=\frac{c_{0}^{2}}{2 R_{S}}$ and to a force $F_{o}=\frac{m c_{0}^{2}}{2 R_{S}}$ with 
a test mass $m$. On the shell this force is equal to the inner force $F_{i}=\frac{G M_{S} m}{R_{S}{ }^{2}}$ which leads to

$$
\frac{c_{0}^{2}}{2}=\frac{G M_{S}}{R_{S}}
$$

This is equation (1). Together with equation (11) the Hubble constant has the easy form

$$
H_{0}=\frac{c_{0}}{\sqrt{2} R_{S}}
$$

New precise and different measurements of the Hubble constant lead to the so called Hubble tension. On the one hand there are the Planck data which lead to a Hubble constant of $67.4 \pm 0.5 \mathrm{Km} /(\mathrm{s} \times \mathrm{Mpc})[(10)]$. On the other hand measurements of the late universe by Riess et al. yield a Hubble constant $H_{0}=74.03 \pm 1.42 \mathrm{Km} /(\mathrm{s} \times \mathrm{Mpc})[(11)]$. That means measurements of the late universe make sense to use when the radius of the shell has to be found. Together with the Hubble constant $H_{0}$ and equation (13) the sphere has a radius of

$$
R_{S} \approx 8.9 \times 10^{25} \mathrm{~m}
$$

The mass of the sphere filled with effective mass particles is (see equation (11))

$$
M \approx 5.99 \times 10^{52} \mathrm{Kg}
$$

The Hubble constant can also be written in the following way (with the Euclidian metric)

$$
H_{0}=\sqrt{\frac{G M}{R_{S}^{3}}}=\sqrt{\frac{4 G \pi \rho_{S F}}{3}}
$$

This leads to a density $\rho_{S F}$ of the scalar field, which is two times the critical density

$$
\rho_{S F}=\frac{3 H_{0}^{2}}{4 G \pi}=2 \rho_{c}=2 \frac{3 H_{0}^{2}}{8 \pi G}
$$

Taking into account the Hubble constant $H_{0}$ the universe would have an age of $t_{0}=\frac{1}{H_{0}} \approx 4.2 \times 10^{17} \mathrm{~s}$. Thus during these 13.32 billion years the light would have traveled a distance of $1.3 \times 10^{26} \mathrm{~m}$. This distance is bigger than $R_{S}$ and a sign that the Hubble constant had a smaller value in the earlier days. In Section 5 we show that this model leads to the lower value of $67.4 \pm 0.5$ $\mathrm{Km} /(\mathrm{s} \times \mathrm{Mpc})$ during the production of the cosmic microwave background, when taking into account a polarization effect. 


\section{Vacuum Energy and the Casimir Effect}

Now we have all the tools to derive the Casimir pressure for two plates in vacuum with the scalar field in the background and without assuming any zero point energy of the vacuum.

The pressure $p$ leading to the Hubble constant has the form $p=\rho c^{2}$. We are only interested in the pressure of one particle with its effective mass. Thus we divide this term by $N$. The value of $N$ is not important because the term will later be reduced. For the next calculations the density $\rho_{S F}=2 \cdot \rho_{c}$ (equation (17)) is used:

$$
p=\frac{\rho_{S F} c^{2}}{N}=\frac{2 \rho_{c} c^{2}}{N}=\frac{3 H_{0}{ }^{2} c^{2}}{4 N G \pi}=\frac{3 c^{2} M}{4 \pi N R^{3}}
$$

As we saw before the mass $\mathrm{M}$ consists of the effective masses $m_{\text {eff }}$ and we can write $M=N \times m_{e f f}$. Together with the value $x$ in section 2 it can be written $M=N \times x \times M_{P}$ and together with $c=c(R)$ and $\hbar=M_{P} c_{0} L_{P}$ this leads to

$$
p=\frac{6 c_{0}^{2} x M_{P} L_{P}}{4 \pi R^{4}}=\frac{3 \times 2 c_{0} x \hbar}{4 \pi R^{4}}=\frac{3 \times 2 c_{0} x \hbar}{4 \pi R^{3} R}
$$

The volume of a sphere $V_{S}=\frac{4}{3} \pi R^{3}$ does not make sense when we talk about two plates in distance to each other with a volume $V_{P}=R^{3}$. By reducing the term by $\frac{4}{3} \pi$ and by reducing the 2 , since we calculate the pressure per plate, the result is

$$
p=\frac{c_{0} \times x \times \hbar}{R^{4}}
$$

When we compare this to the well-known Casimir force for two plates

$$
p_{C}=\frac{c_{0} \pi^{2} \hbar}{240 R^{4}}
$$

there is a difference of $\frac{\pi^{2}}{240}=0.04112$ compared to $x=0.03979=\frac{1}{8 \pi}$. It would be interesting if one of the equations (20) or (21) can be derived as part of a theory of quantum gravity, showing that one of these two numbers (or both numbers) is (are) an approximation. This model also implies that the Casimir effect would not exist without the scalar field.

The dynamical Casimir effect describes the production of photons by undergoing relativistic motion of a mirror. It is explained by virtual photons 
which are converted into observable real photons [(12)]. When the Casimir effect is explained by the scalar field and without vacuum energy this effect has to be explained by the scalar field too. It is natural to assume that our photons with this very low effective speed of light get carried away by the undergoing relativistic motion of the mirror. Due to their previous very low frequency (see Section 5) the mirror frequency is imprinted on the photons. In addition to the detection of real photons two-mode-squeezing is detected [(13)]. This cannot by explained by the carry-away-process. Since we still talk about a scalar field set up by photons of Hawking radiation the source for the entanglement can be the Hawking radiation itself. In an analogue black hole entanglement was already detected [(14)]. In Section 5 we make this aspect clearer.

\section{Hubble Tension and Dark Matter}

Shortly after the Big Bang high temperatures and high field strength appeared. It has been shown that these high field strengths lead to the generation of particle-antiparticle pairs, indicating that the vacuum starts to behave nonlinearly at about $1.3 \times 10^{18} \mathrm{~V} / \mathrm{m}$. Now photons provoke the generation of electron-positron pairs. In $[(4)]$ the energy density for this vacuum breakdown is calculated to $\rho_{E M}=3.1075 \times 10^{25} \mathrm{~J} / \mathrm{m}^{3}$. This is quite similar to the energy density $\rho_{e} \approx 2.374 \times 10^{22} \mathrm{~J} / \mathrm{m}^{3}$ which is needed for the pair production of electrons and positrons. During these high energy densities there should not be any scalar field around because these photons with effective mass would just react in some way. That means by starting with the Planck density $\rho_{P M}=5.155 \times 10^{96} \mathrm{Kg} / \mathrm{m}^{3}$ for the big bang and ending with for example the energy density $\rho_{E M}$ we can calculate a sphere with its radius $R_{e}$ in which there should be no scalar background field and thus no period of expansion of the universe due to the Hubble constant in (11). For the mass density we calculate

$$
\rho_{M E M} \approx \frac{3.1075 \times 10^{25} \mathrm{~J} / \mathrm{m}^{3}}{c_{0}{ }^{2}}=3.46 \times 10^{8} \mathrm{Kg} / \mathrm{m}^{3}
$$

The expansion of the universe reduces the radiation density by $\propto \frac{1}{r^{4}}$. That means when the universe grew to a size of $r_{E M} \approx 7.7 \times 10^{21} \mathrm{~m}$ the scalar field reached its complete form. The size of the universe was around $r_{C M B} \approx$ $4 \times 10^{21} \mathrm{~m}$ when the cosmic microwave background was produced and thus in the similar area compared to typical cosmological length scales. 
By using the spherical model in equation (10) again, this means the sphere has a hollow space in the middle (substracting a smaller sphere of a bigger sphere, see figure 1). Since the fields in the hollow space are eliminating each other, there has not been any expansion due to a positive Hubble constant by any scalar field until the generation of the CMB. From that moment on the normal matter realizes the mass density around the hollow sphere. Thus some adjustments have to be made for the sphere.

In figure 1 the sphere with a hollow space is presented. The radius $R_{S}$ is the radius of the whole sphere. The hollow space has the radius $a \times R_{S}$ and our test mass has the distance to the center of $b \times R_{S} . a$ and $b$ are smaller than 1 and $b>a$. How to calculate the gravitational field at $b \times R_{S}$ in detail is described in Appendix B. Here we go on with the result. The gravitational field at $b \times R_{S}$ is

$$
g_{b}=\frac{G M}{R_{S}^{2}} \times \frac{b^{3}-a^{3}}{\left(1-a^{3}\right) b^{2}}
$$

$M$ is the mass of the complete sphere. We define $k=\frac{b^{3}-a^{3}}{\left(1-a^{3}\right) b^{2}}$ and thus we can rewrite the Hubble constant of equation (11)

$$
H_{0}=\sqrt{\frac{G M}{R_{S}{ }^{3}}}=\sqrt{\frac{G M}{R_{S}{ }^{2}} \times \frac{1}{R_{S}}}
$$

Since the field in equation (23) looks the same, we can define $H_{0 H}=\sqrt{\frac{G M}{R_{S}{ }^{2}} \cdot \frac{k}{R_{S}}}$ and for $k$ we get

$$
k=\frac{H_{0 H}^{2} R_{S}^{3}}{G M}=0.84=\frac{b^{3}-a^{3}}{\left(1-a^{3}\right) b^{2}}
$$

Here the Hubble constant $H_{0 H}=67.4 \mathrm{Km} /(\mathrm{s} \times \mathrm{Mpc})$ for the early universe is used. The term for $k$ can be solved when $a<<b$. Then the result is $\frac{b^{3}}{b^{2}}=b=0.84$. Thus the radius is $0.84 \times 8.9 \times 10^{25} \mathrm{~m}=7.48 \times 10^{25} \mathrm{~m}$. This was around $2.5 \times 10^{17} \mathrm{~s}$ or 7.9 billion years after the Big Bang and fits well with the moment when the universe started to expand accelerated. The approximation $a<<b$ is reasonable because the beginning of section 5 shows that the scalar field got stronger for $r>10^{21} \mathrm{~m}$ and thus $10^{21} \mathrm{~m}<<7.49 \times 10^{25} \mathrm{~m}$. That means when the density of normal matter at some time became that low that it was overtaken by the density of the scalar field / the density of dark energy (if we want to call it that way) the Hubble constant had the 
value of $H_{0 H}=67.4 \mathrm{Km} /(\mathrm{s} \times \mathrm{Mpc})$. From that moment on the value for the Hubble constant grew and reached the value $H_{0}=74.03 \mathrm{Km} /(\mathrm{s} \times \mathrm{MPc})$. Since the universe reached or will soon reach (future measurements of the Hubble constant will show) the shell of the sphere, the Hubble constant will drop by $H_{x}=\sqrt{\frac{G M}{r^{2}} \cdot \frac{1}{R_{S}}}$ with $r>R_{S}$. Here we use the equation for the gravitational field outside a sphere.

During the first 380.000 years the universe was too hot to realize a stable scalar field and the photons had characteristics to typical photons. For the photon in equation (7) we can change the mass $M$ by the mass for the Schwarzschild radius $M=\frac{R_{C M B} c_{0}^{2}}{2 G}$ and end up with

$$
E_{\text {Photon }}=\frac{\hbar c_{0}}{4 \pi R_{C M B}}
$$

For $R_{C M B}$ we use the radius below equation $(22) R_{C M B}=4 \times 10^{21} \mathrm{~m}$. Thus the photons which have been created until 380.000 years after the Big Bang had quite impressive low energies down to $10^{-30} \mathrm{eV}$. Since they act as normal photons they are attractive to gravitation and thus show similar characteristics than fuzzy dark matter. The photon frequency is really low (the wavelength really high) and around $\omega=\frac{c_{0}}{4 \pi R_{C M B}} \approx 6 \times 10^{-15} \mathrm{~Hz}$ (see equation (26)). Then the wavelength is $\approx 10^{22} \mathrm{~m}$. It is important to say that the energy was higher (and thus the wavelength lower) after the big bang, when dark matter created the first mass accumulations with baryonic matter around it. When $R \approx 1 \mathrm{~m}$, the energy was around $10^{-27} \mathrm{~J}=10^{-9} \mathrm{eV}$. The mean energy of the photons is calculated in the following way:

$$
\overline{E_{\text {Photon }}}=\frac{1}{R_{C M B}-2 L_{P}} \int_{2 L_{P}}^{R_{C M B}} \frac{\hbar c_{0}}{4 \pi} \frac{1}{R} d R
$$

This leads to $\overline{E_{\text {Photon }}} \approx \frac{\hbar c_{0}}{4 \pi R_{C M B}}\left(\ln \left(R_{C M B}\right)-\ln \left(2 L_{P}\right)\right) \approx 8.1 \times 10^{-47} J=$ $5.1 \times 10^{-28} \mathrm{eV}$.

If we average the photon energy until $R_{S}$, this leads to $2.4 \times 10^{-32} \mathrm{eV}$. This is equivalent to the mass $m_{P} \approx 4.3 \times 10^{-65} \mathrm{~g}$ and quite similar to the quantized mass $m_{P}=(h / c)(\Lambda / 3)^{1 / 2} \approx 2 \times 10^{-65} \mathrm{~g}$ in [(15)]. It is the same value for $R_{x} \approx 1.95 \times 10^{26} \mathrm{~m}$. When we use this radius as the Schwarzschild radius in equation (1), as we have done before for $R_{S}$, we end up with the mass 
$M_{x} \approx 1.3 \times 10^{53} \mathrm{Kg}$. This is the second important mass in [(15)] for the upper end of the scale. That means with our model we create a connection between quantizing masses at both ends of the scale. If our model is correct, the factor 2 is missing for the small quantized mass and it has to be: $m_{P}=2 \times(h / c)(\Lambda / 3)^{1 / 2} \approx 2 \times 2 \times 10^{-65} g \approx 4 \times 10^{-65} \mathrm{~g}$ and the factor $1 / 2$ is missing for the big mass and it has to be $m_{E}=(1 / 2) \times\left(c^{2} / G\right)(3 / \Lambda)^{1 / 2} \approx$ $(1 / 2) \times 1 \times 10^{56} \approx 5 \times 10^{55} \mathrm{~g}$ in $[(15)]$.

Since it may be interpreted as the quantum of mass, the scalar field tends to define the quantum of mass leading to something similar than a cosmological constant on large scales, where the effects for destroying the scalar field are weakened. Wesson opened the discussion that the constituents are in causal contact (in higher dimensions). That would indicate that the particles creating the scalar field are in causal contact, which gets clearer when we recap that these particle are Hawking radiation, which is entangled. The entanglement also makes the horizon problem in the next section less problematic.

\section{Alternative to inflation theory}

Inflation theory solves these main problems: the flatness problem, the horizon problem, the problem of density fluctuations and the problem of magnetic monopoles. In the following we show how and if our model can solve these problems.

Flatness Problem: We start with the following equation from [(16)]:

$$
\dot{\epsilon}=(1+\epsilon) \epsilon \frac{\dot{a}}{a}(1+3 \omega)+2 \frac{\dot{c}}{c} \epsilon
$$

Here $\epsilon=\Omega-1$ with $\Omega=\rho / \rho_{c}$ and $\omega$ is the energy density. One way to solve the flatness puzzle is that the speed of light is decreasing, thus $\left(\frac{\dot{c}}{c}\right)<0$. Then $\epsilon$ strives towards 0 and the flatness problem is solved for early times because then $\Omega$ strives towards 1 and the density strives towards the critical density. If there is some kind of sharp phase transition with $|\dot{c} / c|>>\dot{a} / a$ then the expansion term in (28) can be neglected and $\dot{\epsilon} / \epsilon=2 \dot{c} / c$, which leads to $\epsilon \propto c^{2}$. To see if our model can solve this problem we use equation (4) for the speed of light and change $R_{S}$ by $c_{0} t$ with the time $t$ because we are looking for the first moments after the Big Bang and the expansion takes place with the speed of light. Thus we get

$$
c(t)=\sqrt{\frac{2 L_{P} c_{0}}{t}}
$$


and

$$
\dot{c}(t)=\frac{1}{2} \sqrt{\frac{2 L_{P} c_{0}}{t^{3}}}
$$

Thus $|\dot{c} / c|=\frac{1}{2 t}$. Since the Big Bang starts around Planck time with $t_{P} \approx$ $10^{-44} \mathrm{~s}$, then $|\dot{c} / c|>>\dot{a} / a$ is fulfilled and the flatness problem solved.

For later times around $t_{0}$ we saw that $\rho_{S F}=2 \rho_{c}$ (equation (17)). This process towards $2 \rho_{c}$ started after the generation of the CMB because then the normal matter entered the area of the sphere with constant density of the effective mass particles. Today the density for normal matter and dark matter shifts further downwards and is overtaken by the density $\rho_{S F}$. Thus we take the approximation $\rho \approx \rho_{S F}=2 \rho_{c}$. That means $\Omega=2$. We use the term

$$
H^{2}(\Omega-1)=k \frac{c^{2}}{R^{2}}
$$

Here $k$ is the curvature. With $H_{0}^{2}=\frac{G M}{R_{S}^{3}}=\frac{c^{2}}{R_{S}^{2}} \frac{G M}{R_{S} c^{2}}$ we know that $k=\frac{G M}{R_{S} c^{2}}=$ 0.5 in the near future. This goes on until the normal mass passed the outer shell of the sphere. Then, as we see in figure ..., the density $\rho_{S F}$ will drop with the dropping Hubble constant $H \propto 1 / r$ for $r>R_{S}$.

Horizon problem: Since the scalar field, which was generated during the collapse of the last local universe, acts as a background field in the new universe we live in, there was already a structure when the Big Bang began. These background photons have been in thermal interaction for such a long time that the field is completely homogeneous. Furthermore, during the expansion and collapse of the predecessor universe light had so much time to expand space-time and to flatten the space-time structure. And this process will go on. The matter/energy compressed in the black hole was in direct contact to each other until the black hole exploded. Thus this matter is already homogeneous.

Problem of density fluctuations: Inflation theory solves this problem by quantum fluctuations which are inflated during the inflation phase. In our model everything including the background field is completely homogeneous except the Big Bang explosion itself. When we assume that the predecessor black hole explodes at the end of its live time macroscopic and inhomogeneous structures are thrown to the surroundings and act as condensation cores for the freed up dark matter. Future detailed studies have to show if 
this idea is compatible with the CMB.

Magnetic Monopoles: This model has no clue for solving the problem of magnetic monopoles. For the big bang and the generation of our universe magnetic monopoles are not necessary. At one point they have been important, but this moment can be e.g. four or even ten universe lifetimes ago. When we assume that one universe in the oscillating history started from nothing they must have been important.

\section{Scenario}

The complete scenario is shown in figure 2. The figure shows the time from the black hole in the predecessor universe to the beginning compressing phase in our universe. On the y-axis the energy densities and the Hubble parameter are shown. We start with the predecessor universe. After it collapsed to a black hole the evaporation process took place. The black hole emitted Hawking radiation and the Schwarzschild radius shrank. During the shrinking process the scalar field was set up (see Section 2). How long did it take that the black hole evaporated completely? Since our model includes a constant evaporation process due to the constant effective mass and a quantum hypothesis for the mass of black holes, first we calculate the time for one effective mass to evaporate $[(17)]$ :

$$
\tau^{*}=\frac{5120 \pi G^{2} m_{\text {eff }}^{3}}{\hbar c_{0}^{4}} \approx 10^{-44} s
$$

In the next step we calculate the number $N$, which describes of how many effective masses the black hole consists:

$$
N=\frac{M}{m_{e f f}}=\frac{5.9928 \times 10^{52} \mathrm{Kg}}{8.6598 \times 10^{-10} \mathrm{Kg}}=6.92 \times 10^{61}
$$

Then the lifetime of the black hole can be calculated:

$$
\tau \approx N \times \tau^{*}=6.92 \times 10^{61} \times 10^{-48} s \approx 10^{18} s
$$

Thus it took about 35 Billion years. During that time the scalar field density grew and more or less the whole energy, which was absorbed by the black hole before, was shifted to the environment. At the end of its lifetime the scalar field density was extremely high and similar to the Planck density. Now the 
core of the black hole exploded (which introduced the Big Bang) and the photons in the scalar field act as dark matter (see Section 5) and the typical attenuation of radiation density and matter density happened (see figure 2). This was possible because due to equation (4) the photon speed was nearly $c_{0}$ at a Schwarzschild Radius of $\approx L_{P}$. The radiation moved with the speed of light and started a chain reaction with the high density scalar field. This high density scalar field appeared because the Hawking radiation rate had a constant flux, but with a smaller volume $\rho_{S F} \propto \frac{1}{r^{3}}$ the density grew massive. The chain reaction went on and more and more effective mass particles became dark matter or radiation and baryonic matter later. This happened as we see in Section 5 until the fluctuations in the scalar field became less. The photons stayed in the background grid and since the time dependent variations aimed towards zero or a small value the pressure became negative and from this moment on the Hubble constant pushed the universe apart. This happened during the generation of the cosmic microwave background. When the matter density became less than the density of the scalar field particles the universe had a Hubble constant of about $67.4 \frac{\mathrm{Km}}{s \times M p c}$. This Hubble constant grew due to the hole in the middle of the sphere without scalar field (see Section 5) and reached its value today of $74.03 \frac{K m}{s \times M p c}$. Depending on where we are on the sphere the Hubble constant will still grow and later decrease or directly decrease (see figure 2). Then the attraction due to gravitation will get stronger and mass density will rise until everything is compressed to a new black hole and the process will go on again. Since there is always the circumstance that not all photons are attracted by the new rising black hole, the entropy of the whole process grows. The entropy of the black hole is derived in Appendix C.

\section{Conclusion}

We showed a phenomenological approach for a cycling universe without cosmological constant and vacuum energy density in its original meaning. Our vacuum energy density is the heritage of a scalar field of the predecessor universe, which was generated by Hawking radiation during the black hole evaporation. In our model the Hubble constant came into play after the generation of the CMB with the value of $67.4 \frac{\mathrm{Km}}{s \times M p c}$. This value rise to the value $74.03 \frac{\mathrm{Km}}{s \times M p c}$. In the future the Hubble constant will decrease (or first still increase and then decrease) because the scalar field has a maximum volume and by leaving the sphere, that delimits the scalar field spatially, the 
Hubble constant will decrease and the gravitation will win the upper hand, leading to a black hole, which will lead to the process beginning again. This model can solve the flatness and horizon problem with its phenomenological approach and future studies will show if this can also be done in detail. To verify or falsify this model future more precise measurements of the Hubble constant are necessary.

\section{Appendix A}

Average photon velocity in sphere with radius $R_{S}$ (see figure 1 ):

$$
\overline{c\left(R_{S}\right)}=\frac{1}{R_{S}-2 L_{P}} \int_{2 L_{P}}^{R_{S}} c_{0} \sqrt{\frac{2 L_{P}}{R_{S}}} d R_{S} \approx \frac{2 c_{0} \sqrt{2 L_{P}}}{R_{S}}\left(\sqrt{R_{S}}-\sqrt{2 L_{P}}\right)
$$

For a Schwarzschild blackhole (no electric charge, no angular momentum) with a Schwarzschild radius of $R_{M W}=1.2 \times 10^{10} \mathrm{~m}$, which is the Schwarschild radius of $\mathrm{SgrA}^{*}$ in our milky way, the average photon velocity is $\overline{c\left(R_{M W}\right)} \approx$ $3 \times 10^{-14} \mathrm{~m} / \mathrm{s}$.

For the sphere with the radius $R_{S}=8.9 \times 10^{25} \mathrm{~m}$ in equation (14) the average photon velocity is $c\left(R_{S}\right)=3.6 \times 10^{-22} \mathrm{~m} / \mathrm{s}$.

\section{Appendix B}

Gravitational field inside a sphere with a hollow sphere in its middle (see figure 1):

For calculating the gravitational field we first calculate the reduced mass density and the reduced volume. The reduced mass density is defined by

$$
\rho^{*}=M / V=\frac{M}{(4 / 3) \pi R^{3}-(4 / 3) \pi(a R)^{3}}=\frac{M}{(4 / 3) \pi\left(R^{3}-(a R)^{3}\right)}
$$

Here $M$ is the mass and $R$ the radius of the whole sphere. $a \times R$ is the radius of the inner sphere. For the volume we can write

$$
V^{*}=(4 / 3) \pi(b R)^{3}-(4 / 3) \pi(a R)^{3}=(4 / 3) \pi\left(b^{3} R^{3}-a^{3} R^{3}\right)
$$


Here $b \times R$ is the radius of our test mass / observer. We can use this radius here because only the volume until this distance from the center is necessary. Now the reduced mass can be calculated

$$
M^{*}=\rho^{*} V^{*}=\frac{M\left(b^{3} R^{3}-a^{3} R^{3}\right)}{R^{3}-a^{3} R^{3}}
$$

The gravitational field at $b \times R$ is

$$
g=G \frac{M^{*}}{b^{2} R^{2}}=\frac{G M}{R^{2}} \frac{\left(b^{3}-a^{3}\right)}{\left(1-a^{3}\right) b^{2}}
$$

\section{Appendix C}

To calculate the entropy of the black hole we use equation (26) and extend it by the Schwarzschild radius $R_{S}$

$$
E_{\text {Photon }}=\frac{\hbar c_{0} R_{S}}{4 \pi R_{S}^{2}}=\frac{\hbar c_{0} R_{S}}{A}
$$

Here the black hole surface is $A=4 \pi R_{S}^{2}$. The equivalent mass $m_{P}=\frac{E_{P h o t o n}}{c_{0}^{2}}$ is

$$
m_{P}=\frac{\hbar R_{S}}{A c_{0}}
$$

We use equation (1) and end up with

$$
m_{P}=\frac{2 G \hbar M}{A c_{0}^{3}}
$$

Since at some point all the mass $M$ of the black hole is evaporated, we write $M=N \times m_{P}$ and for $N$ we get

$$
N=\frac{A c_{0}^{3}}{2 \hbar G}
$$

Since the helicity of photons can only have two different values, the entropy for all photons and thus for the black hole is $S=N k_{B} \ln 2$ with the Boltzmann constant $k_{B}$. Together with equation (43) this leads to

$$
S=\frac{A c_{0}^{3} k_{B}}{\hbar G} \frac{\ln 2}{2}
$$


When we compare this with the Bekenstein Hawking entropy $S_{B H}=\frac{A c_{0}^{3} k_{B}}{4 \hbar G}$ the distinction is $\frac{\ln 2}{2} \approx 0.35$ compared to $1 / 4=0.25$. This distinction has maybe the same reason as the one for equation (20) and (21). There the difference is much lower but it is in a completely different range. There two plates in vacuum are described and here a black hole is described. Thus the difference can maybe suffer the effect of summation for other regimes.

\section{References}

[1] J. D Bekenstein, Lett. Nuovo Cimento, 11, 467 (1974).

[2] J. D Bekenstein, V. Mukhanov, Phys.Lett. B360, 7 (1995).

[3] G. Dvali, C. Gomez, S. Mukhanov, (2011) [arXiv:1106.5894[hep-ph]].

[4] E. Margan, Estimating the vacuum energy density - an overview of possible scenarios, Experimental Particle Physics Department, Jozef Stefan, Institute, Ljubljana, Slovenia.

[5] S. W. Hawking, Commun.Math.Phys., 43, 199-220, (1976).

[6] A. Almheiri, et al., Journal of High Energy Physics, 149, (2020).

[7] J. Steinhauer, nature physics, 12, 959-965 (2016).

[8] R. R. Caldwell, R. Dave, P. J. Steinhardt , Phys. Rev. Lett, 80, 15821585, (1998).

[9] S. W. Hawking, Nature, 248, 30-31, (1974).

[10] Planck Collaboration, N. Aghanim, et al., (2018) [arXiv:1807.06209[hep$\mathrm{ph}]$.

[11] A. G. Riess, et al., (2019) [arXiv:1903.07603[hep-ph]].

[12] S. A. Fulling, M. P. C. W. Davies, Proceedings of the Royal Society A, 348, 393 (1976).

[13] C. M. Wilson, G. Johansson, A. Pourkabirian, J.R. Johansson, T. Duty, F. Nori, P. Delsing, nature, 479, 376-379 (2011).

[14] J. Steinhauer, Nature Physics, 12, 959-965 (2016). 
[15] P. S. Wesson, Mod. Phys. Lett. A19 (2004) 1995.

[16] J. Magueijo, Reports on Progress in Physics. 66. 10.1088/00344885/66/11/R04 (2003).

[17] M. Lopresto, The Physics Teacher, 41, 299-301 (2003). 